\title{
High-throughput magnetic particle washing in nanoliter droplets using serial injection and splitting
}

\author{
William Stephenson ${ }^{*}$ (])
}

\begin{abstract}
Droplet microfluidics has emerged as a promising technique to perform high-throughput, massively-parallel chemical and molecular biological reactions. Droplet microfluidic operations such as droplet generation, sorting, and fluid addition are well established; however, fluid exchange (i.e. washing) at high-throughput is challenging to implement. Here we present a microfluidic device architecture that utilizes wash buffer injection preceding a splitting junction in proximity to a magnetic field to transfer paramagnetic microparticles across a concentration gradient within a single droplet. The device can operate at high throughput $(50 \mathrm{~Hz})$ while preserving input droplet volume at the collection outlet as verified using high speed imaging. Using a two-stage device, combined microparticle retention rates (up to $97.5 \%$ ) and high wash efficiency (92.9\%) is demonstrated using dye absorbance and fluorescence. This method can be performed in a serial array to obtain an arbitrary degree of wash efficiency and integrated into lab-on-a-chip systems for use in multi-step microfluidic bioassays or single-cell genomic applications requiring high-fidelity washing steps within droplets.
\end{abstract}

Keywords: Droplet microfluidics, Magnetic particle washing

\section{Background}

Droplet microfluidics involves sample partitioning into nanoliter or smaller volumes and has been extensively used in a variety of fields including biology, medicine, chemistry and physics [1-4]. Most commonly, aqueous fluids are encapsulated into droplets surrounded by a continuous phase of an oil surfactant mixture. Droplet-based sample partitioning into miniscule volumes allows for faster reaction kinetics due to decreased diffusion times compared to bulk scale reactions [5]. The ability to generate droplets at $\mathrm{kHz}$ frequency permits massively parallel reaction schemes with precision control of reaction volumes due to the highly monodisperse nature of microfluidic droplet generation. A major goal of microfluidics is to mimic bench-level fluid manipulations within the droplet format in order to take advantage of smaller reaction volumes, massively-parallel scalability, compartmentalization and decreased reagent use.

*Correspondence: wstephenson@nygenome.org

Technology Innovation Lab, New York Genome Center, New York, NY 10013, USA
Magnetic and non-magnetic oligonucleotide barcoded microparticles have recently been used in conjunction with droplet microfluidic or microwell partitioning to profile the surface proteomes and or transcriptomes of thousands of cells in a massively parallel manner [6-13]. Magnetic microparticles are used extensively in molecular biology and genomics as versatile systems for genomic immunoassays, cell capture and sorting, and nucleic acid binding and size selection [14-16]. Their ease-of-use, high efficiency and low cost have made magnetic microparticles a ubiquitous tool and the solid support of choice for washing and capture at the bench-top and in high throughput robotic protocols. In order to translate standard molecular biology reactions using magnetic microparticles into the droplet format and to expand the repertoire of high-throughput single cell genomic applications, magnetic microparticles should be seamlessly integrated into on-chip droplet assays. The majority of onchip droplet microfluidic assays can be described by primary unit operations which include droplet generation, fluid injection, sorting, splitting and washing. Additive functions such 
as generation and injection are commonplace, however subtractive functions such as washing are less established, especially in a robust manner at high throughput $[1,17,18]$.

Washing magnetic beads in droplets on digital microfluidic (DMF) devices using electrowetting on demand (EWOD) based platforms is straightforward; typically an electromagnet is activated beneath an array of electrode pads which are used to manipulate droplets containing magnetic particles [19]. DMF and EWOD platforms are easily automated, however they are inherently limited in throughput due to constraints of fabricating and addressing large arrays of electrode pads each of which can accommodate at most one droplet. In light of this limitation, continuous flow droplet washing approaches have been developed to increase throughput. Magnetic tweezers have been used to controllably immobilize magnetic particles derived from a droplet within a section of tubing [20,21]. This approach showed extremely high recovery of magnetic microparticles and excellent wash efficiency, however the throughput was severely limited due to active switching of the magnetic tweezers electromagnet. Additionally, the magnetic tweezers apparatus is large and not amenable to miniaturization and integration into microfluidic systems. Another approach involves the synchronization of two droplet trains followed by electrocoalescence and splitting [22]. One droplet train contained magnetic particles in a background solution to be washed away, the other droplet train contained wash buffer into which the magnetic particles are transferred. The droplet trains are forced through a rail-road network of channels to equilibrate pressure and synchronize the positions and velocities of the droplets. Droplets are then electrocoalesced at a junction proximal to a stationary magnetic field, thereby transferring magnetic microparticles into the wash buffer droplet prior to physical splitting of the electrocoalesced droplet into two daughter droplets. In spite of relatively efficient washing in a single step, multiple failure mechanisms were observed and throughput was low due to the required synchronization of droplet trains in the rail-road network.

In order to realize a fully integrated droplet lab-on-a-chip system that operates at high throughput it is important to address the challenges associated with magnetic microparticle based droplet microfluidic washing. Here we outline a method to perform magnetic microparticle washing in nanoliter droplets using electrocoalescence based injection [23], magnetic microparticle margination, and splitting. In this implementation these operations all occur in the same region nearly simultaneously to achieve efficient washing of magnetic microparticles into droplets with near equivalent volume to the input droplets. This approach favors injection over droplet synchronization based washing techniques in order to improve throughput significantly. Injection by this method additionally introduces an in-droplet concentration gradient, where the injected solution effectively displaces the original droplet contents to the side of the droplet distal to the injection point. Injection occurs under the influence of an externally applied magnetic field from a chip-embedded stationary magnet, to immediately transfer magnetic microparticles from the input fluid into the injected wash buffer. After injection and transfer of magnetic microparticles the droplet is split via a microchannel bifurcation immediately downstream of the injection junction. Optimal placement of the splitting junction ensures that the majority of the droplet volume in the retained droplet comprises the injected solution and not the input solution. These three modalities, wash buffer injection, magnetic particle transfer, and immediate splitting, operate in concert and form a method for high efficiency washing of magnetic microparticles within droplets at high throughput. This platform can be arranged in serial to obtain an arbitrary level of wash efficiency. Here we demonstrate a two-stage device consisting of inject-transfer-split junctions where the collection of the first wash step is ported directly into the input of the second wash step (Fig. 1). This platform is amenable to miniaturization in lab-on-a-chip formats and has potential for use in high-throughput droplet based magnetic microparticle bioassays requiring efficient wash steps.

\section{Methods}

\section{Device design and fabrication}

Dark field photolithography transparency masks (Advance Reproductions, North Andover, MA) were designed in AutoCAD 2016. Silicon wafers were cleaned with piranha solution for $30 \mathrm{~min}$ at room temperature. SU-8 3050 was spin coated to a thickness of $100 \mu \mathrm{m}$ and exposed through the transparency mask using a Karl Suss MJB3 mask aligner. After development, microstructures were used in soft lithography PDMS molding. After PDMS slabs were cut from the wafer, inlet and outlet ports were punched using a $1 \mathrm{~mm}$ biopsy punch. Next, three square regions $\sim 3.5 \mathrm{~mm}^{2}$ were cut from the slab to accommodate three square $\left(1 / 8^{\prime \prime} \times 1 / 8^{\prime \prime}\right)$ grade N52 neodymium magnets (B222G-N52, $\mathrm{KJ}$ magnetics). Next the PDMS slab was bonded to a glass slide with oxygen plasma for $45 \mathrm{~s}$ and heat cured for $10 \mathrm{~min}$ in an oven at $75{ }^{\circ} \mathrm{C}$. Next, Aquapel (Pittsburgh glass works Pittsburgh, PA) was perfused through droplet channels and dried at $75{ }^{\circ} \mathrm{C}$ oven for $10 \mathrm{~min}$. Next, $50 \mathrm{mM}$ (3-mercaptopropyl)trimethoxysilane (MPTMS) in acetonitrile was perfused through electrode array channels for $30 \mathrm{~s}$ and purged. The device was then placed in the oven for $10 \mathrm{~min}$ at $75^{\circ} \mathrm{C}$. Next, The device was placed on a hotplate and heated to $215^{\circ} \mathrm{C}$ for $10 \mathrm{~min}$. Using rubber tipped tweezers a short strip $(\sim 5 \mathrm{~mm})$ of $0.030^{\prime \prime}$ diameter 52In/48Sn low melting temperature solder (Indium Corporation, Clinton, NY) was forced into the electrode array channels pre-treated with MPTMS. After all electrode array channels were filled with solder, the device was removed from the hotplate using wafer tweezers 


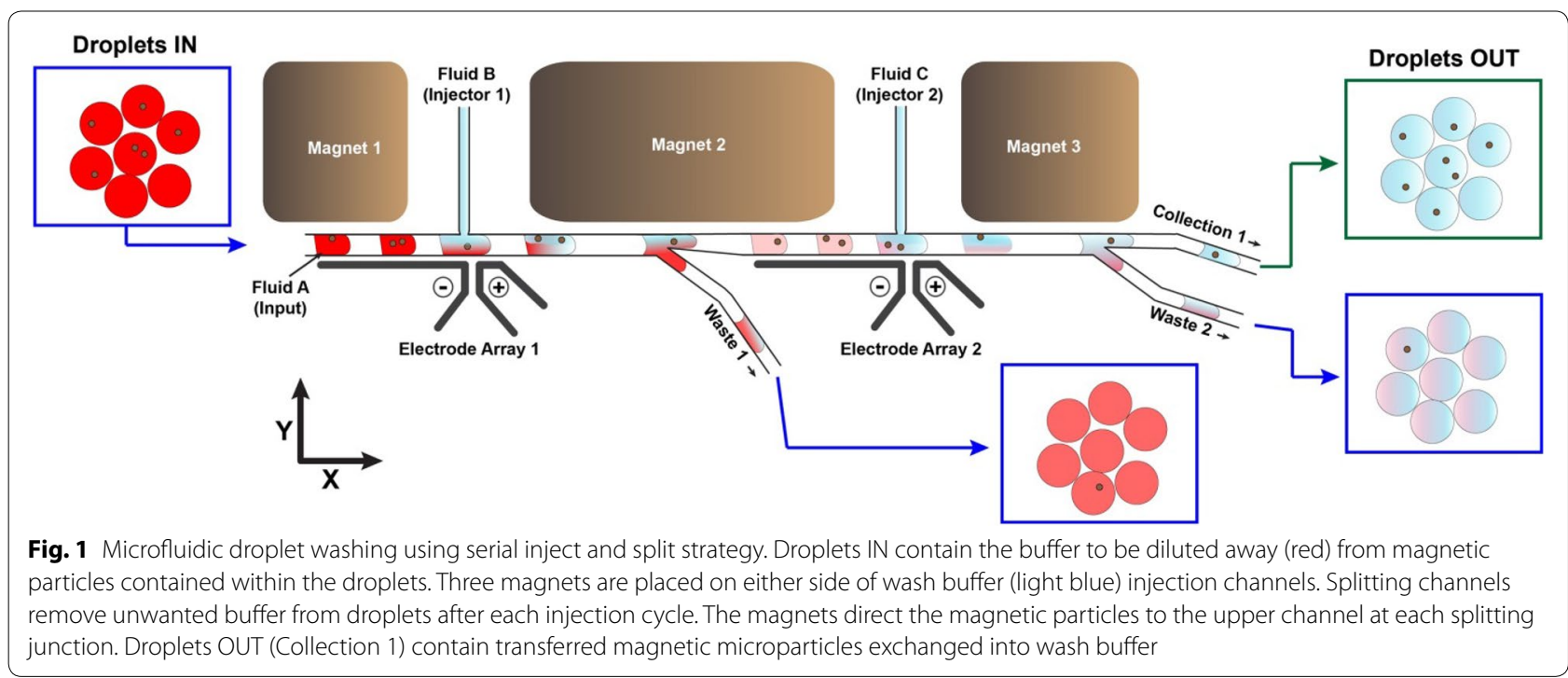

and $60 \mathrm{~mm}$ copper wire segments obtained from stripping and separating 28 gauge copper wire (AlphaWire, Elizabeth, $\mathrm{NJ}$ ) were placed into the electrode array ports using the rubber tweezers. Finally, the device was cooled $(10 \mathrm{~min})$ and epoxy was used to cover the fragile copper/metal electrode array contacts and let set overnight.

\section{Experimental setup and device operation}

Devices were operated using an inverted microscope at $4 \times$ and $10 \times$ magnification and videos were imaged using a high-speed camera (FASTCAM mini AX200 Photron, Tokyo, Japan) at 5000 FPS. Electrode array contacts were linked and connected to a high-voltage fluorescent light bulb inverter (CXA-L0505-NJL, TDK Corporation) powered at $5 \mathrm{~V}$. Syringe pumps were used to flow fluorinated oil at $325 \mu \mathrm{l} / \mathrm{h}$ and magnetic particles of varying diameter and magnetic type $(1 \mu \mathrm{m}$ superparamagnetic, $1 \mu \mathrm{m}$ paramagnetic, $5 \mu \mathrm{m}$ paramagnetic, and $22 \mu \mathrm{m}$ paramagnetic, Spherotech Inc.) suspended in $1 \times$ PBS at $180 \mu \mathrm{l} / \mathrm{h} .22 \mu \mathrm{m}$ diameter magnetic particles were suspended using a PTFE coated winged magnetic stir raft (773W-5,9, V\&P scientific) and stirred using a magnetic stirrer (710D2, V\&P scientific). Flow from injection channels was obtained by pressurizing the head space of custom made vials with microtubing connections (New England Small Tube). Pressure was monitored using two independent pressure sensors (Honeywell, Trustability 015PGAA5) connected to regulators (R-800-90W/K, Airtrol Inc.), one for each injection channel in the twostage device. Operating pressures for the injection channels were 1.3 PSI and 1.1 PSI for the first and second channels respectively. Pressure actuation was controlled through solenoid valve manifold (Festo) in LabView via a DAQ (National Instruments, X Series USB-6343).

\section{Analysis}

For optimization experiments bromophenol blue was used as a visual aid and calibration solution for input buffer removal. Bromophenol blue dye was imaged under a compatible band pass filter FWHM $=10 \pm 2 \mathrm{~nm}$ (FB590-10, Thor Labs Inc.). Fluorescein salt was also used as a visual aid for washing efficiency. Serial dilutions of bromophenol blue were generated and absorbance spectra measured on NanoDrop 2000 spectrophotometer for each experiment for calibration purposes. For droplet washing efficiency experiments, emulsions were broken using $1 \mathrm{H}, 1 \mathrm{H}, 2 \mathrm{H}, 2 \mathrm{H}$-perfluoro1 -octanol and the absorbance of the aqueous portion was measured. Droplets were visualized in FuchsRosenthal hemacytometers (InCyto). High-speed videos were analyzed using droplet morphometry and velocimetry (DMV) software, generously supplied by Amar Basu. Post processing and additional data analysis was performed in MATLAB (MathWorks).

\section{Results and discussion Working principle}

Our device consists of a droplet generation region to encapsulate magnetic microparticles into droplets followed by two inject, transfer, split junctions arranged in serial (Fig. 2a, b). The droplet generation rate was measured using high speed imaging and was typically on the order of $50 \mathrm{~Hz}$. Three N52 neodymium magnets were arranged such that magnets occupy both sides of each injection channel. Magnets arranged upstream of injection channels served to pre-orient magnetic microparticles and reduce transfer times after injection and before splitting. Waste channels were designed to have equal fluidic resistance to their counterpart collection channels. 

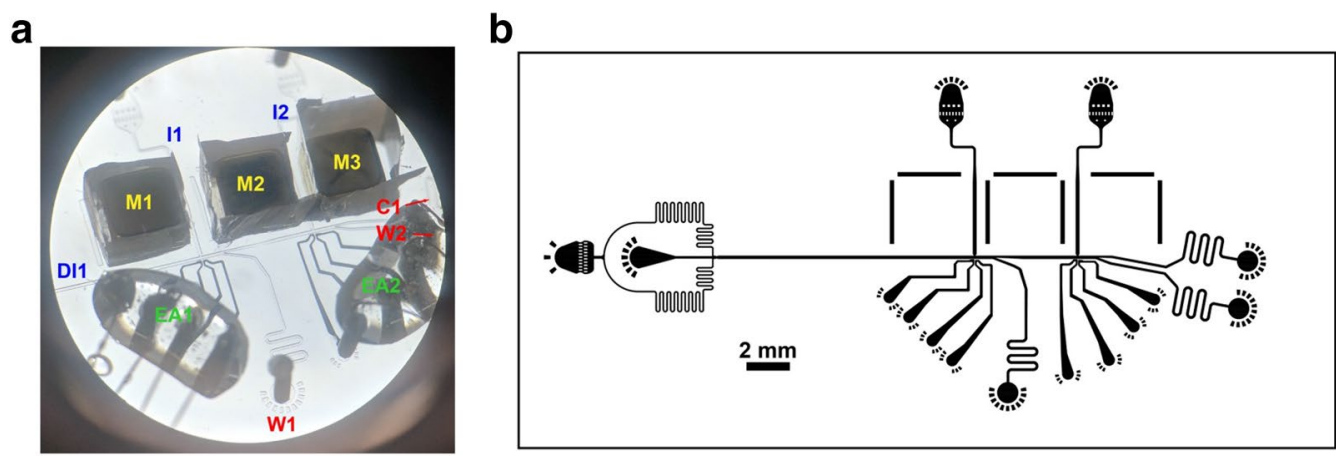

Fig. 2 Microfluidic device and schematic. a Microscope view of the microfluidic chip. The input channels are marked in blue (Droplet Input 1 - DI1, Injection 1—11, and Injection 2-12) and the outlet channels are marked in red (Waste 1—W1, Waste 2-W2, and Collection 1—C1). Three N52 magnets (M1, M2, M3) are labeled in yellow. The electrode arrays are labeled in green (EA1, EA2). b Microfluidic photolithography mask design for the two-stage washing device

The distance between the injection junction and the splitting location was carefully optimized for the flow rates used. If the distance is too long the injected wash buffer will mix via diffusion and convection within the travelling droplet, decreasing wash efficiency which relies on a wash buffer concentration gradient within the droplet. If the distance is too short the magnetic particles may not have sufficient time to traverse the droplet axis after experiencing a downward force due to the injection of pressurized wash buffer. The force on the magnetic microparticles depends on a variety of factors. The magnetic force on the microparticles is calculated by

$$
F_{m a g}=\frac{1}{2} \frac{\gamma V \Delta \chi}{\mu_{0}} \nabla B^{2}
$$

where $\mathrm{V}$ is the volume of the magnetic microparticle and $\gamma$ is the fraction of the microparticle volume occupied by magnetic material (i.e. the magnetic core), $\Delta \chi$ is the net magnetic susceptibility of the microparticles in a medium, $\mu_{0}$ is the permittivity of free space and $B$ is the magnetic field. The magnetic microparticles also experience a Stoke's drag force calculated by

$$
F_{\text {drag }}=-6 \pi r \eta v
$$

where $r$ is the hydrodynamic radius of the microparticle, $\eta$ is the viscosity of the medium, and $v$ is the velocity of the microparticle. The sum of these two forces is equal to zero:

$$
F_{\text {mag }}+F_{\text {drag }}=0
$$

from which the velocity of the magnetic particle(s) can be calculated by

$$
v_{m a g}=\frac{\gamma V \Delta \chi}{12 \pi r \eta \mu_{0}} \nabla B^{2} .
$$

The volume fraction of magnetic material (magnetic core) for the microparticles used in this study is estimated to be $15 \%(\gamma=0.15)$ according to the manufacturers specifications. The magnetic field strength of the neodymium magnet was determined from the topographic field description provided by the manufacturer (Additional file 1: Figure S1). The expected velocity of a microparticle within the magnetic field encountered here is highly dependent on the size of the microparticle and its distance from the magnet (Fig. 3a). An estimate of the condition for efficient recovery of magnetic microparticles is dependent on the transit time of the droplet between the injection point and splitting junction and the transit time of the magnetic particle(s) from the lower portion of the droplet just after injection to the upper portion of the droplet prior to splitting:

$$
t_{\text {drop }} \geq t_{\text {mag }} \text {. }
$$

Transit times for both the droplet and the magnetic particle(s) are dependent upon the flow rate (i.e. droplet velocity), the distance from the injection channel to the splitting junction and the type/size of magnetic microparticles used:

$$
\frac{d}{v_{\text {drop }}} \geq \frac{|\delta W-y|}{v_{\text {mag }}}
$$

where $d$ is the distance from the injection site to the splitting junction, $v_{d r o p}$ is the flow-rate dependent droplet velocity, $\delta$ is the ratio of the waste channel width to the sum of the waste and collection channel widths at the splitting junction (daughter droplet splitting fraction), $W$ is the width of the droplet in the microchannel, $y$ is the position of the magnetic microparticle along the $y$-axis immediately after injection relative to the top of the 

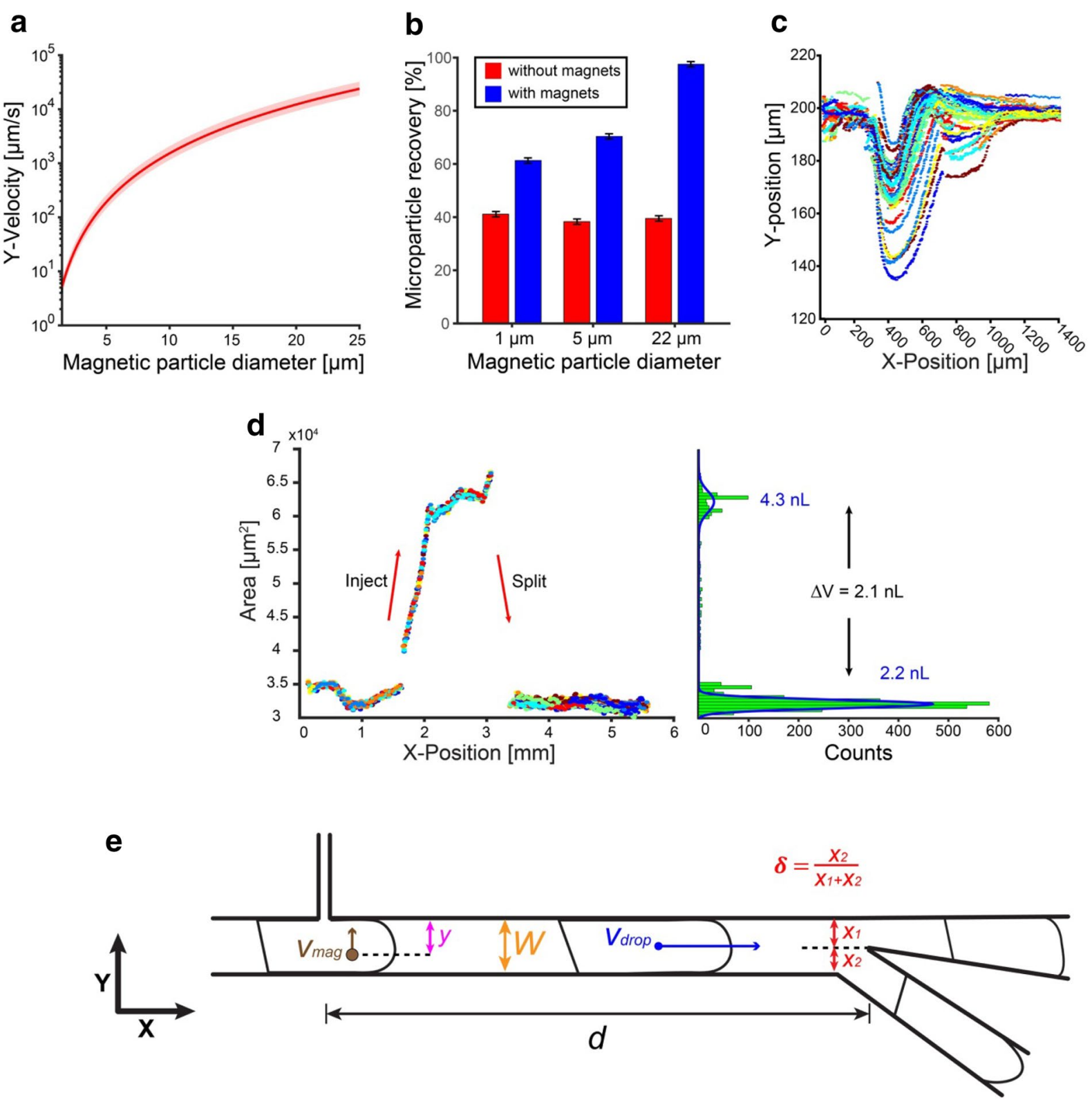

Fig. 3 Magnetic particle characteristics and droplet volume preservation. a Theoretical microparticle velocity in the Y-direction as a function of microparticle diameter according to Eq. (4). The shaded region around the curve indicates the change in distance between the microparticle and the stationary magnet as the microparticle traverses through the channel immediately after injection. $\mathbf{b}$ Microparticle recovery under standard flow rates in the two-stage washing microfluidic chip. c High speed video tracking of $22 \mu \mathrm{m}$ diameter microparticles in the injection region. $\mathbf{d}$ High speed video tracking of droplets during wash buffer injection and subsequent splitting into daughter droplets. e Schematic of critical device geometries and operating parameters for efficient recovery of magnetic particles described in Eq. (6)

droplet and $v_{\text {mag }}$ is the velocity of the magnetic particle(s) derived in Eq. (4).

\section{Magnetic microparticle recovery and droplet volume preservation}

We assessed the microparticle recovery as a function of three paramagnetic microparticle sizes 1,5 , and $22 \mu \mathrm{m}$ in diameter. For the optimal flow rates determined the observed recovery (fraction of magnetic microparticles recovered in the final collection outlet compared to the combined waste channels) was most efficient (97.5\%) for the largest diameter microparticles $(22 \mu \mathrm{m})$ (Fig. 3b). This is due to the larger magnetic core and resulting higher velocity in the magnetic field. Smaller diameter $(\sim 1 \mu \mathrm{m})$ superparamagnetic particles were also tested, but due to higher net magnetic susceptibilities and resulting higher 
velocities, the injection channel required modifications to achieve efficient recovery (Additional file 2: Video S1). This represents a limitation of the current device design, which may need to be tailored to the properties of the magnetic particles used in the droplet based assay. Because high speed imaging and tracking of larger diameter particles is less challenging than for smaller particles, the $22 \mu \mathrm{m}$ diameter magnetic microparticles were used for the remainder of proof-of-principle experiments in this study. In the absence of a magnetic field adjacent to the injection channels, collective recovery for all microparticles was approximately $40 \%$ presumably due to the force imparted through injection (Fig. 3b) which biases additional microparticles to the waste outlet channels during splitting. Using high-speed imaging the trajectories of individual $22 \mu \mathrm{m}$ microparticles were tracked using the DMV software [24] and superimposed (Fig. 3c). The injection induced Y-axis deflection is typically less than $70 \mu \mathrm{m}$ and particles return to the top portion of the droplet within about $500 \mu \mathrm{m}$ distance travelled in the $\mathrm{X}$ direction. The distance between the injection channel and the splitting junction is $1 \mathrm{~mm}$ for the proof-ofprinciple devices used in this study indicating that higher throughput is achievable. Using high speed imaging the velocity of the particles was tracked just after injection. Typically maximum velocities did not exceed $2500 \mu \mathrm{m} / \mathrm{s}$ in fair agreement with the expected value (Eq. 4). Slight deviations from the expected velocity may be due to microparticle and magnetic core size dispersity. In addition, the placement of the stationary magnet varies slightly from device to device causing small variations in the magnetic field gradient experienced by the microparticles. In order to verify preservation of droplet volume after splitting, high-speed video of the injection and splitting cycle was acquired. Droplet area was tracked prior-to and during injection and after the bifurcation channel. Droplet area is seen to increase linearly during injection followed by an abrupt decrease at the splitting junction as the two daughter droplets are tracked separately. The area was integrated along the channel height to obtain the volume change for the injection-splitting cycle. Injection and splitting cycle traces were superimposed and compiled into a histogram. The histogram was best fit by two Gaussian distributions indicating identical input droplet and output daughter droplet volumes of $2.2 \mathrm{nl}$ upon injection of $2.1 \mathrm{nl}$ and equal volume splitting (Fig. 3d).

\section{Magnetic microparticle washing efficiency}

In order to verify that the operations of injection of wash buffer, magnetic microparticle transfer, and splitting could operate simultaneously we generated droplets from a solution containing bromophenol blue and quantified the effect of washing by measuring absorbance $(590 \mathrm{~nm})$ of individual droplets. Using the two-stage washing device we directly observed a decrease in input droplet buffer concentration as a decrease in droplet absorbance at the collection outlet (Fig. 4). Simultaneously the magnetic microparticle is visualized being deflected due to wash buffer injection pressure and returns to the top portion of the droplet after about $25 \mathrm{~ms}$. Following particle

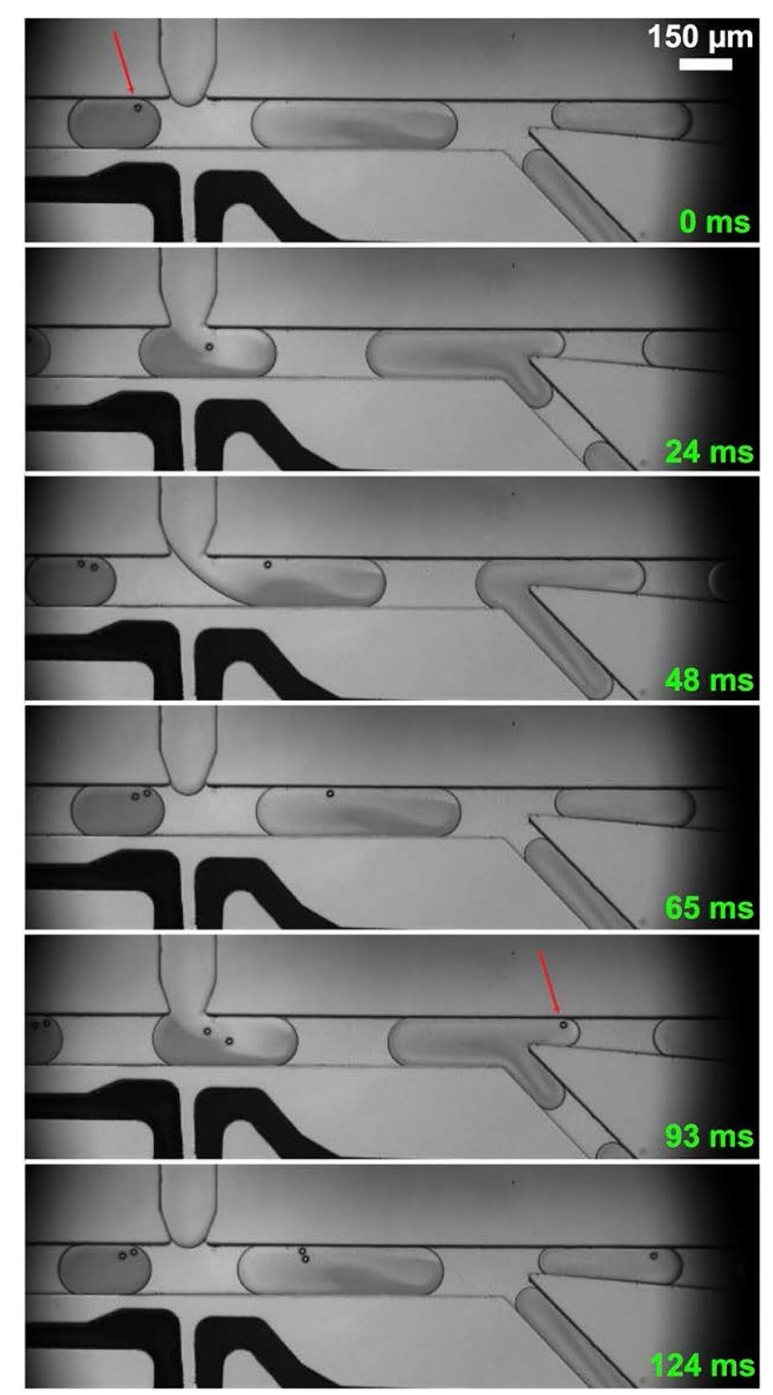

Fig. 4 Snapshots from high speed video of droplet washing. Incoming droplets with high bromophenol blue concentration (dark) are traveling from left to right. The magnetic microparticle is indicated with a red arrow. Droplets encounter the injection channel across from electrodes. Upon injection (24 ms) the microparticle is deflected downward due to the injection pressure. At $48 \mathrm{~ms}$ the microparticle has recovered its position at the top portion of the droplet under the influence of a magnetic field from a stationary magnet out of view. Finally the droplet is split into two daughter droplets: the one to be collected (top channel) contains the magnetic microparticle in a lower concentration of bromophenol blue 

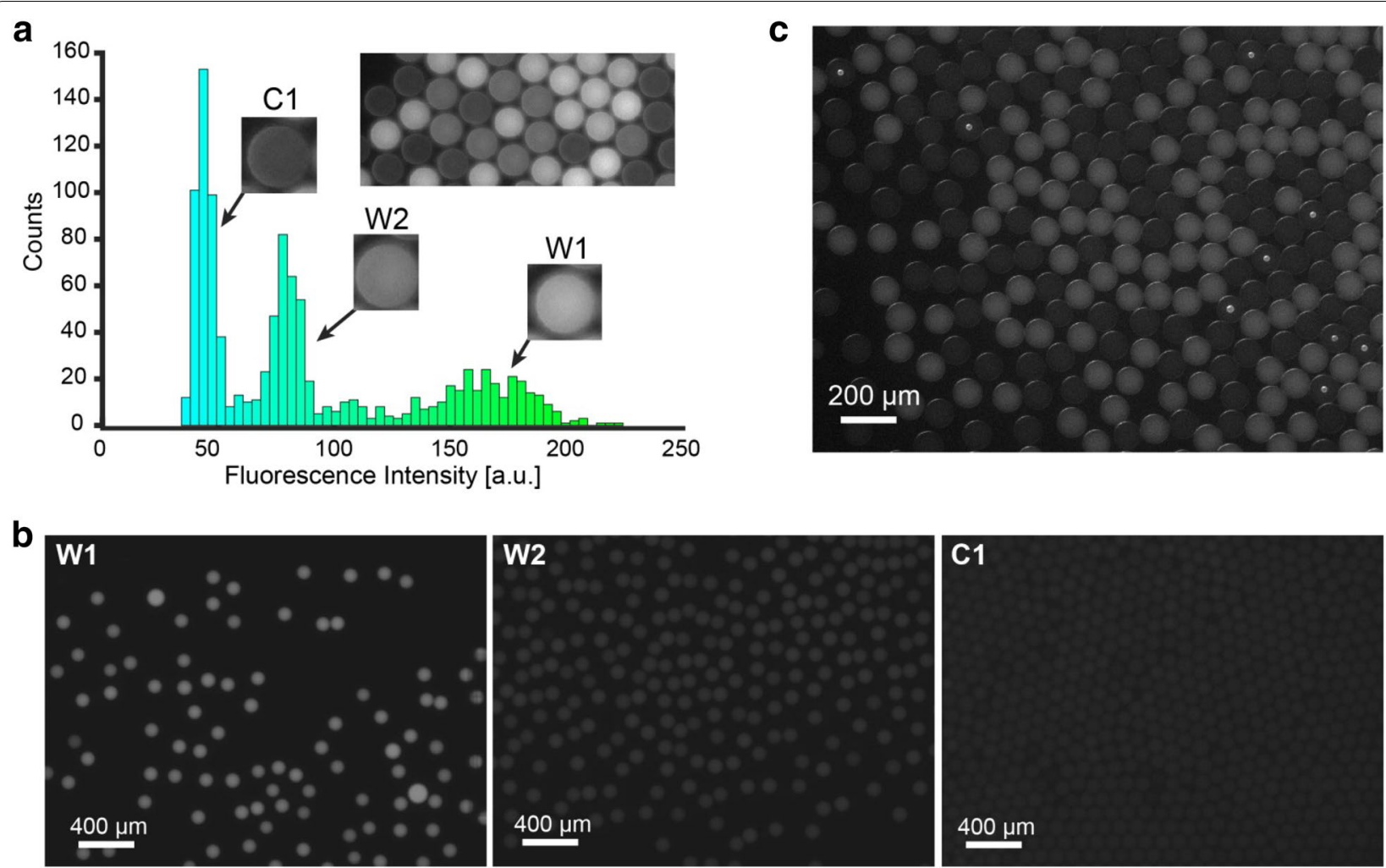

Fig. 5 Fluorescence measurements of droplet concentration after washing. a Droplets from outlets W1, W2, and C1 were pooled from the two-stage device, imaged and fluorescence intensity recorded. b Fluorescence images of droplets collected individually from outlets W1 (left), W2 (center), and C1 (right). c Droplets from outlets W1, W2 and C1 pooled from a two-stage device in an experiment with $22 \mu$ m diameter magnetic microparticles in the input droplets. The auto-fluorescent magnetic microparticles are present only in droplets with low solution fluorescence

margination the droplet is split such that the collection daughter droplet (right top channel) has a visibly lower absorbance compared to the waste daughter droplet (right bottom channel) (Fig. 4 and Additional file 3: Video S2). The entire two-stage serial washing process can be observed in Additional file 4: Videos S3 and Additional file 5: S4 showing electrocoalescence induced injection, magnetic microparticle transfer, and droplet splitting and decrease in dye concentration for ease of visualization. In addition to bromophenol blue we used fluorescein to visualize the effectiveness of washing. Input droplets containing $10 \mathrm{mM}$ fluorescein in 1X PBS were washed in the two-stage device and the outlets of W1, W2, and C1 channels were imaged (Fig. 5a, b). Three primary distributions of fluorescence were measured corresponding to each of the outlets from the two-stage device. (The intensity of input droplet fluorescence of $10 \mathrm{mM}$ fluorescein saturated the microscope camera on this scale, so input droplets were omitted from this analysis) After verification of droplet dilution through the serial injection array, we included magnetic microparticles to assess whether microparticle margination could be achieved simultaneously with dilution to achieve effective washing and retention of microparticles. Droplets from outlets W1, W2 and C1 were pooled from the two-stage device and imaged (Fig. 5c). The magnetic microparticles were observed to be present in the least fluorescent droplets indicating recovery of particles into washed or buffer exchanged droplets only.

In order to more directly quantify the washing efficiency a calibration curve was determined from a set of known bromophenol blue dye concentrations and peak absorbance at $590 \mathrm{~nm}$ (Fig. 6a, b). The two-stage device was used to wash and collect droplets before breaking the droplets and measuring the absorbance of the aqueous component of the emulsion. Peak intensities for each outlet (W1, W2, and C1) can be seen to progressively decrease indicating removal of bromophenol blue dye from droplets. The peak intensities were measured and the calibration curve was used to derive effective concentrations of each droplet outflow (Fig. 6c). The bromophenol blue dye concentrations of input and collected droplets were 500 and $35.5 \mathrm{nM}$ respectively, corresponding to a $92.9 \%$ wash efficiency or a 15 -fold reduction in concentration of input solution (Fig. 6d). Comparable approaches achieved a 25 -fold reduction in concentration 


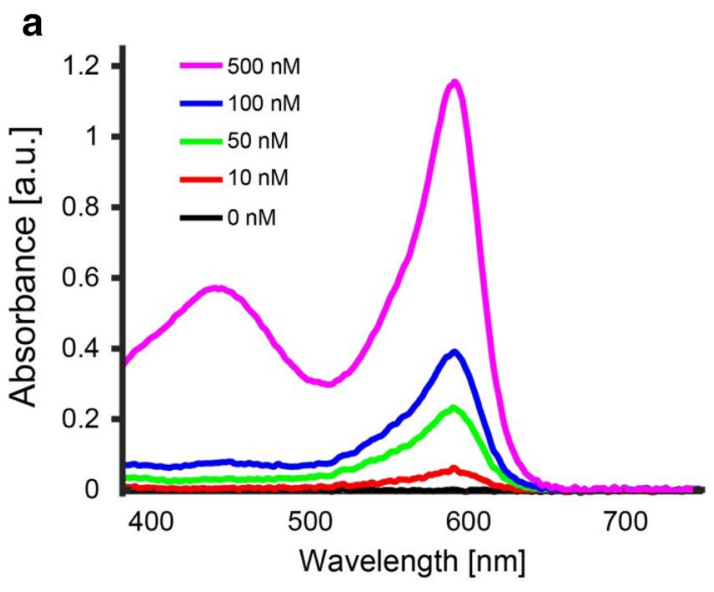

C

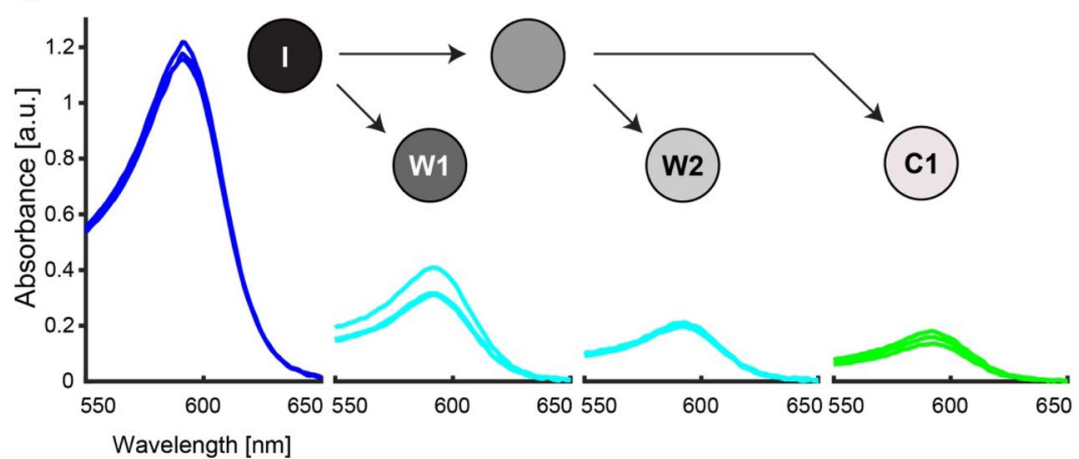

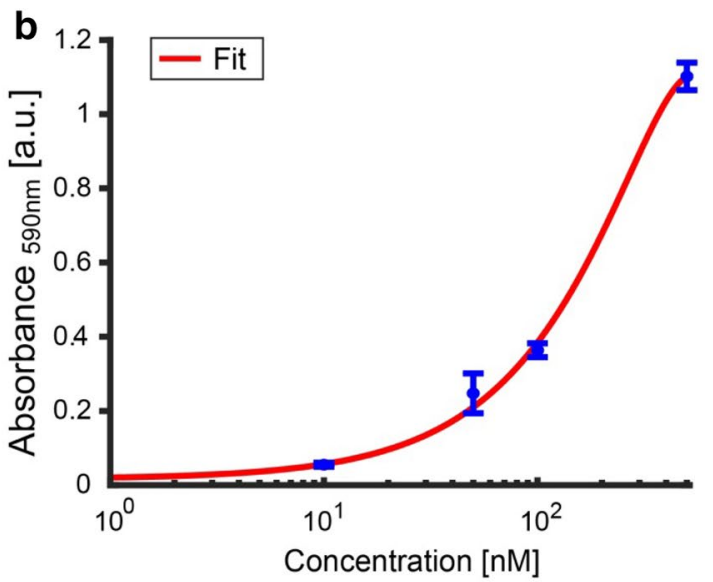

d

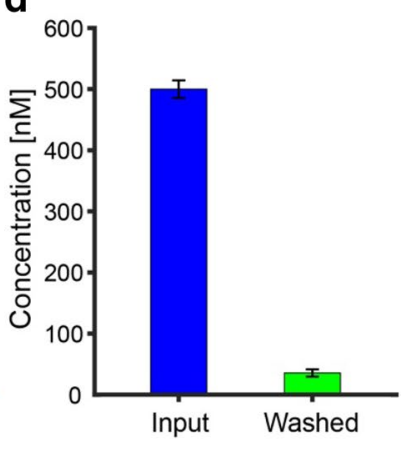

Fig. 6 Dye based measurements of droplet concentration. a Visible absorbance spectra from bromophenol blue dye. b From the absorbance spectra a calibration curve can be established to measure unknown concentrations between 500 nM and about 10 nM. c Bulk absorbance spectra in triplicate derived from washed droplets (I-input, W1 — waste 1, W2 — waste 2, C1 — collection 1). d Wash concentration change as determined from $590 \mathrm{~nm}$ bromophenol blue absorbance and the calibration curve in $\mathbf{b}$

of input solution albeit at much lower throughput $\sim 2 \mathrm{~Hz}$ and with multiple reported failure mechanisms [22]. Wash efficiency in this work could be improved through a number of approaches. The geometry of the microfluidic device, such as distance between the injection point and splitting junction (parameter $d$, Fig. 3e) in addition to the splitting fraction $\delta$, could be carefully optimized to minimize the amount of diffusion immediately after was buffer injection. This is highly dependent on magnetic particle properties and the flow rate. In this proof-ofprinciple study we deliberately kept the parameter $d$ large as a conservative measure, however further optimization by decreasing $d$ would result in higher wash efficiency due to less time for diffusive mixing to occur between the waste portion and the injected wash buffer portion of the droplet prior to splitting. Finally, the nature of the microfluidic device described here allows for an arbitrary number of modules to be arranged serially to obtain strict wash efficiencies which may be required for sensitive bioassays.

\section{Conclusions}

We have presented a robust method to wash magnetic microparticles within droplet microfluidic systems at high throughput using a serial array of injection channels. Using high speed imaging, absorbance and fluorescence measurements the wash efficiency was determined to be $92.9 \%$ while retaining $96 \%$ of $22 \mu \mathrm{m}$ magnetic microparticles. Smaller diameter microparticles could be implemented with minor alteration to the microfluidic device, namely the injection channel geometry and the margination channel length prior to droplet splitting. Magnetic microparticles in this study were washed in droplets at high throughput $(\sim 50 \mathrm{~Hz})$ representing a tenfold improvement in throughput over alternative approaches with comparable wash efficiency. Further flow rate optimization will allow for even higher throughput washing. The serial nature of this flexible method allows for 'daisy-chaining' multiple wash modules for applications requiring strict high-fidelity washing. Importantly, this microfluidic device does not rely on bulky off-chip 
instrumentation for magnetic microparticle manipulation and is amenable to miniaturization. This device is a step towards robust high-throughput microparticle washing for lab-on-a-chip systems and can be used to complement the droplet microfluidic unit operations. We envision this device can be integrated into microfluidic workflows for the development of complex droplet based bioassays or used in single-cell genomic applications requiring high-throughput washing of captured analytes of interest.

\section{Additional files}

Additional file 1: Figure S1. Magnetic field strength from stationary magnet. The magnetic field strength as a function of distance was derived from the manufacturers topographic field strength representation and fit using an exponential sum. The gradient of the magnetic field ( $y$-axis) as a function of distance derived from the fit established.

Additional file 2: Video S1. High-speed video of modified device for washing of superparamagnetic microparticles. In order to prevent magnetic microparticle transfer into the injection channel, the channe diameter was decreased and was oriented at a $\sim 45^{\circ}$ angle with respect to the input droplet train channel. Droplet generation frequency is $50 \mathrm{~Hz}$, magnetic microparticle size is $\sim 1 \mu \mathrm{m}$.

Additional file 3: Video S2. High-speed video of two-stage droplet washing device. Input droplets generated at $50 \mathrm{~Hz}$ travelling from left to right contain a high concentration of bromophenol blue dye. $22 \mu \mathrm{m}$ magnetic microparticles are seen loaded into droplets. Droplets are injected and magnetic microparticles are marginated to the upper half of the droplet before splitting. As the microscope pans right, the second stage is encountered and the process is repeated.

Additional file 4: Video S3. High-speed video of stage one of droplet washing device. Input droplets generated at $50 \mathrm{~Hz}$ travelling from left to right contain a high concentration of bromophenol blue dye. $22 \mu \mathrm{m}$ magnetic microparticles are seen loaded into droplets. Droplets are injected and magnetic microparticles marginate to the upper half of the droplet before splitting. The two daughter droplets are seen to have different absorbance indicating removal of dye from the input droplet.

Additional file 5: Video S4. High-speed video of two-stage droplet washing device with overloaded dye. Input droplets generated at $50 \mathrm{~Hz}$ travelling from left to right contain a very high concentration of bromophenol blue dye. Droplets are injected and dye concentration is decreased significantly prior to splitting. As the microscope pans right, the second stage is encountered and the process is repeated.

\section{Authors' contributions}

WS conceived of the method, fabricated devices, performed experiments, analyzed the data, and wrote the manuscript. The author read and approved the final manuscript.

\section{Acknowledgements}

We gratefully acknowledge financial support from the New York Genome Center and technical support from Kunal Pandit and critical reading of the Manuscript by Peter Smibert, in addition to support from members of the Technology Innovation Lab at the New York Genome Center. We also thank Professor Amar Basu (Wayne State University) for developing and providing the Droplet Morphometry Velocimetry (DMV) software.

\section{Competing interests}

The author declares no competing interests.

\section{Availability of data and materials}

The datasets supporting the conclusions of this article are included within the article and its additional files.

\section{Funding}

This study was supported from funding through the New York Genome Center.

\section{Publisher's Note}

Springer Nature remains neutral with regard to jurisdictional claims in published maps and institutional affiliations.

Received: 16 May 2018 Accepted: 18 June 2018

Published online: 21 June 2018

\section{References}

1. Guo MT, Rotem A, Heyman J, Weitz D (2012) Droplet microfluidics for high-throughput biological assays. Lab Chip 12:2146

2. Xu J et al (2017) Controllable microfluidic production of drug-loaded PLGA nanoparticles using partially water-miscible mixed solvent microdroplets as a precursor. Sci Rep 7:1-12

3. Gach PC, Iwai K, Kim P, Hillson N, Singh AK (2017) Droplet microfluidics for synthetic biology. Lab Chip 17:3388-3400

4. Beatus T, Bar-Ziv RH, Tlusty T (2012) The physics of 2D microfluidic droplet ensembles. Phys Rep 516:103-145

5. Song H, Chen DL, Ismagilov RF (2006) Reactions in droplets in microfluidic channels. Angew Chem 45:7336-7356

6. Macosko EZ et al (2015) Highly parallel genome-wide expression profiling of individual cells using nanoliter droplets. Cell 161:1202-1214

7. Klein AM et al (2015) Droplet barcoding for single-cell transcriptomics applied to embryonic stem cells. Cell 161:1187-1201

8. Zheng GXY et al (2017) Massively parallel digital transcriptional profiling of single cells. Nat Commun 8:1-12

9. Stoeckius M et al (2017) Simultaneous epitope and transcriptome measurement in single cells. Nat Methods 14:865-868

10. Peterson VM et al (2017) Multiplexed quantification of proteins and transcripts in single cells. Nat Biotechnol. https://doi.org/10.1038/ nbt.3973

11. Shahi P, Kim SC, Haliburton JR, Gartner ZJ, Abate AR (2017) Abseq : Ultrahigh-throughput single cell protein profiling with droplet microfluidic barcoding. Nat Publ Gr. https://doi.org/10.1038/srep44447

12. Han $X$ et al (2018) Mapping the mouse cell atlas by microwell-seq. Cell 172:1091-1107

13. Stephenson W et al (2018) Single-cell RNA-seq of rheumatoid arthritis synovial tissue using low cost microfluidic instrumentation William. Nat Commun 9:1-10

14. Lee JT, Abid A, Cheung KH, Sudheendra L, Kennedy IM (2012) Superparamagnetic particle dynamics and mixing in a rotating capillary tube with a stationary magnetic field. Microfluid Nanofluidics 13:461-468

15. Lis JT, Schleif R (2000) Size fractionation of double -stranded DNA by precipitation with polyethylene glycol. 2:383-389

16. Trevor LH, O'Connor-Morin T, Aparna R (1994) DNA purification and isolation using a solid-phase. Nucleic Acids Res 22:4543-4544

17. Simon MG, Lee AP (2012) Microfluidic droplet manipulations and their applications. Microdroplet Technol. https://doi. org/10.1007/978-1-4614-3265-4

18. Kaminski TS, Scheler O, Garstecki P (2016) Droplet microfluidics for microbiology: techniques, applications and challenges. Lab Chip 16:2168-2187

19. Sista RRS et al (2008) Heterogeneous immunoassays using magnetic beads on a digital microfluidic platform. Lab Chip 8:2188-2196

20. Ferraro D et al (2016) Microfluidic platform combining droplets and magnetic tweezers: application to HER2 expression in cancer diagnosis. Sci Rep 6:25540 
21. Ali-Cherif A, Begolo S, Descroix S, Viovy JL, Malaquin L (2012) Programmable magnetic tweezers and droplet microfluidic device for high-throughput nanoliter multi-step assays. Angew Chem 51:10765-10769

22. Lee H, Xu L, Oh KW (2014) Droplet-based microfluidic washing module for magnetic particle-based assays. Biomicrofluidics 8:044113

23. Abate AR, Hung T, Mary P, Agresti JJ, Weitz D (2010) High-throughput injection with microfluidics using picoinjectors. Proc Natl Acad Sci USA 107:19163-19166
24. Basu AS (2013) Droplet morphometry and velocimetry (DMV): a video processing software for time-resolved, label-free tracking of droplet parameters. Lab Chip 13:1892-1901

\section{Submit your manuscript to a SpringerOpen ${ }^{\circ}$ journal and benefit from:}

- Convenient online submission

- Rigorous peer review

- Open access: articles freely available online

- High visibility within the field

- Retaining the copyright to your article

Submit your next manuscript at $\boldsymbol{\nabla}$ springeropen.com 